\title{
LAS FAMILIAS ENCABEZADAS POR MUJERES Y LA FORMACIÓN DE NÚCLEOS EXTENSOS: UNA REFERENCIA AL PERÚ
}

\author{
Marta Tienda y Sylvia Ortega Salazar \\ University of Wisconsin Madison \\ Universidad Autónoma Metropolitana
}

\section{Introducción}

UNO DE LOS TEMAS más significativos olvidados por la demografía formal ha sido el análisis de las familias incompletas.* Es decir, núcleos en los cuales debido a la ausencia del padre, la mujer asume el papel y las funciones que corresponden a los jefes de familia. A pesar de que las familias encabezadas por mujeres han existido siempre y de que además en todas las sociedades contemporáneas son numéricamente significativas (Buvinic y Youssef, 1978), no ha sido sino hasta muy recientemente que los científicos sociales han mostrado algún interés en este aspecto de las estructuras familiares.

La mayor parte de los estudios de caso que han abordado el tema de las familias incompletas hacen referencia a la situación particular de los Estados Unidos (Duncan, 1976; Ross y Samhill, 1975; Cooney, 1979; Cutright, 1974; Sweet, 1972) y se han centrado en el análisis de las implicaciones económicas y sociales correlativas a una expansión numérica de este tipo de familias, sobre todo constatable en años recientes. En este sentido, existe una amplia documentación que detecta empíricamente las formas en las que los jefes de familia mujeres se encuentran en desventaja respecto a los jefes varones.

En lo que hace a la significación económica y demográfica de las familias incompletas en América Latina, es muy poco lo que se sabe. Exceptuando los trabajos de Walker y Gendell (1976) sobre Guate-

* Una versión preliminar de este texto se presentó en el Congreso de la Population Association of America, Denver, Colorado. La investigación fue financiada por la Escuela de Graduados, Universidad de Wisconsin-Madison (Proyecto No 190075) y el Departamento de Ciencias de la Agricultura. El trabajo de computación fue apoyado por el Centro de Ecología y Demografía de la misma institución. Agradecemos la asistencia técnica de Mary Miron. 
mala, Merrick (1977) en el Brasil, y Tienda (1980) sobre el Perú, es escasa la literatura demográfica que alude concretamente a las diferencias en la composición de los núcleos familiares que resultan del tipo de jefatura.

A pesar de que la información sobre el tema es.evidentemente muy reducida, una de las cuestiones que se ha convertido casi en un lugar común es la afirmación en el sentido de que los jefes de familia enfrentan serios problemas para satisfacer las necesidades de consumo básico de sus hijos ya que la ausencia de un compañero varón anula las posibilidades de dividir el trabajo interno, lo que resulta en una pérdida de flexibilidad para la unidad familiar en su conjunto. Si bien las mujeres que asumen la jefatura familiar tienden a sumarse a la fuerza de trabajo -sobre todo en los llamados sectores informales de la economía- dando así solución a sus necesidades alimenticias mínimas, lo cierto es que les resulta sumamente difícil cumplir con la doble función de madres de familia y proveedoras de los medios de subsistencia del núcleo (Buvinic y Youssef, 1978; 74-75). Esta situación obliga a que los núcleos incompletos se reestructuren buscando por esta vía compensar la ausencia del padre. Nuestra hipótesis es que la formación de núcleos familiares extensos es un mecanismo que permite a las familias incompletas restablecer su flexibilidad permitiéndoles introducir fórmulas razonables para dividir el trabajo en el interior de la unidad.

Este análisis pretende sustentar la hipótesis enunciada por la vía de determinar las formas concretas bajo las cuales se modifica la composición de las familias peruanas. A este efecto se analiza:

1. si efectivamente el tipo de jefatura familiar ${ }^{1}$ determina en alguna forma la conformación de unidades extensas y

2. cómo varía la propensión hacia la formación de familias extensas según los lugares de residencia y el tipo de jefatura familiar.

A los efectos de ubicar el grado y las formas específicas en las cuales las mujeres jefes de familia se encuentran en desventaja respecto a las cabezas de familia varones, hemos optado por caracterizar a las dos subpoblaciones utilizando para ello algunas variables demográficas y socioeconómicas. Este ejercicio tiene la virtud de ubicar. a las familias encabezadas por mujeres en relación con aquéllas que

1 Por jefatura tradicional entendemos la modalidad de padre y madre donde la cabeza de familia es el varón. 
poseen una jefatura tradicional, al tiempo que hace posible describir los rasgos que distinguen a la subpoblación femenina. En segundo término pasamos a especificar la relación que existe entre el lugar que ocupan las jefes de familia, y los miembros del núcleo en la fuerza de trabajo y la tendencia a la formación de familias extensas. En la medida en que este segundo nivel de análisis incluye asimismo la inspección del comportamiento de dicha relación entre las familias cuya jefatura es la tradicional, permite, eventualmente, determinar en qué medida y por qué causa las familias incompletas presentan una tendencia más aguda hacia la reestructuración de sus propias unidades.

Finalmente, a través de un análisis de clasificación múltiple se desagregan los efectos de las distintas variables seleccionadas a fin de determinar la forma en que inciden sobre las diferentes tendencias a la recomposición observadas en los dos tipos de familias.

La selección del caso peruano como objeto de estudio obedece fundamentalmente a que se trata de un país en el que, como en otras naciones de América Latina, se presenta un período de rápida expansión demográfica que coincide con una serie de cambios sociales y económicos significativos.

\section{Las jefaturas femeninas y la formación de familias extensas: consideraciones teóricas}

Independientemente de su pertenencia de clase, puede afirmarse que las mujeres en general se encuentran en una situación económica y social desventajosa respecto de los hombres. ${ }^{2}$ Esta diferencia, que tiene que ver básicamente con la estructura patriarcal de las sociedades modernas, ha sido generalmente entendida como un efecto imputable a la incapacidad de las economías periféricas para generar suficientes empleos. En los países latinoamericanos la incorporación de la mujer a la fuerza de trabajo se encuentra limitada más que por factores ideológicos ${ }^{3}$ por razones estructurales (Singelmann y Tienda,

2 Así, por ejemplo, en Asia, mientras que el $57 \%$ de las mujeres son analfabetas, un $39 \%$ de la población masculina se encuentra en la misma situación. En el Africa las proporciones son $88 \%$ de las mujeres y el $66 \%$ de los hombres; en los Fstados Arabes la relación es del $89 \%$ para la población femenina y $66 \%$ para la masculina; finalmente, en América Latina, el $30 \%$ de las mujeres son analfabetas y el $25 \%$ de los hombres son tambićn iletrados (Tomados de la Tribuna, No. 6, 2do. trimestre 1980).

En relación al empleo, los datos que a continuación se consignan son igualmente ilustrativos de las desventajas estructurales de la subpoblación femenina: en tanto que dos terceras partes de todas las horas de trabajo son realizadas por la mujer, solamente $\frac{1}{10}$ parte de los ingresc, globales son apropiados por la población femenina. Asimismo, solamente una centésima parte de todas las propiedades del mundo pertenece a la mujer (tomado de La Mujer y el Trabajo, OIT, 1/1978).

3 Como en el caso de los países musulmanes del Medio Oriente. 
1979). Específicamente, el proceso de desarrollo capitalista en el Perú ha involucrado el desplazamiento de una parte considerable de la población que anteriormente se encontraba vinculada a actividades del sector primario. Si bien en forma parcial ésta ha pasado a formar parte de la fuerza de trabajo industrial, lo cierto es que inexorablemente existe un remanente importante, grupo en el que las mujeres en general tienden a estar sobre-representadas (Chaney y Schmenks, 1975).

Aunque en algunos países de América Latina las mujeres han logrado ubicarse en ramas tales como la textil, la electrónica, la industria alimenticia, etcétera y mayoritariamente en los servicios, en el caso particular del Perú, la proporción de mujeres empleadas ha declinado sustancialmente entre 1940 y 1972, período en el que decre ció su participación del $35.4 \%$ al $20.3 \%$. Así, en la medida en que las mujeres en general tienden a ser excluidas de la fuerza de trabajo asalariada, podemos aseverar que la necesidad de implementar estrategias alternativas a fin de garantizar la supervivencia de sus núcleos familiares es sumamente aguda. Es en este contexto en el que la posibilidad de la formación de familias extensas cobra sentido en tanto que alternativa que eventualmente puede garantizar la viabilidad económica de la unidad doméstica. En efecto, la adición de un familiar adulto a la unidad nuclear puede representar un beneficio importante para la familia ya sea que éste contribuya con ingresos monetarios, remplazando así al jefe de familia, o bien que se haga cargo del trabajo doméstico facilitando la incorporación de la mujer jefe de familia a la fuerza de trabajo. En parte, nuestra hipótesis propone que las dificultades específicas que enfrenta la mujer jefe de familia para reconciliar la doble función de proveedora de ingresos monetarios y de madre pueden ser parcialmente superadas a través del mecanismo de recomposición de su familia nuclear, lo que le brinda la posibilidad de compensar la ausencia de un trabajador de tiempo completo, le facilita la captación de ingresos suplementarios o bien le permite asegurar el cuidado de su familia.

Esta propuesta no es del todo novedosa; existen en la literatura demográfica algunos análisis que ilustran cómo las llamadas poblaciones marginales tienden a enfrentar las desventajosas condiciones sociales y económicas que les son características. Quijano (1970) por ejemplo, ha sugerido que las estrategias de supervivencia entre estas subpoblaciones son factibles ya que las familias tienen la capacidad de reorganizarse de forma tal que les resulta posible enfrentar condiciones materiales sumamente restrictivas. Por su parte, Larissa Lomnitz (1975) en su estudio sobre los marginados de la Ciudad de México destaca el papel que juegan las redes familiares de asistencia mutua 
en tanto garantes de la supervivencia de los individuos. El trabajo de Lomnitz deja claro que entre las familias encabezadas por una mujer, cuyas desventajas relativas son aún mayores que las de las familias completas, la dependencia respecto de las formas de asistencia y reciprocidad suplementada por parientes y vecinos, así como la utilización más intensa del trabajo infantil, ${ }^{4}$ son mecanismos que contribuyen a paliar las estrecheces de la unidad.

Los cambios en el nivel de la configuración de la propia estructura familiar son particularmente significativos ya que facilitan el intercambio entre los miembros y pueden ulteriormente elevar los mínimos de bienestar de la familia en su conjunto. Los patrones de reconstrucción familiar pueden asumir la forma de intercambio de asistencia o bien derivar en la captación de miembros vinculados a la fuerza de trabajo. En tanto que la investigación sobre los patrones de reciprocidad familiar ha quedado fundamentalmente en manos de la antropología social (Lomnitz, 1976; Roberts, 1976), la demografía social ha descubierto una veta de análisis en lo que respecta a la especificación de la importancia de los patrones de extensión familiar como medio para aliviar situaciones asociadas a la disrupción marital.

No todas las mujeres que jefaturan sus familias tendrán la misma inclinación a reestructurar sus núcleos; ésta dependerá básicamente de dos factores: en primer lugar, la severidad de su situación económica y en segundo, la disponibilidad de "espacios" vitales..$^{5}$ Esto es,

4 Datos más recientes muestran claramente que los niños mayores sustituyen en las labores domésticas a la jefe de familia mujer cuando ésta trabaja fuera del núcleo. En el cuadro 1 se registran los resultados de una encuesta aplicada en las zonas ruralès de Kenya:

Cuadro 1

SUSTITUCIÓN DE LABORES DEL HOGAR Y ÁREAS RELACIONADAS ENTRE LAS MUJERES VENDEDORAS DE LOS MERCADOS

\begin{tabular}{lccc}
\hline T a r a & $\begin{array}{c}\text { Empleados } \\
\text { Remunerados }\end{array}$ & Niños & $\begin{array}{c}\text { Otros=/ } \\
\text { Familiares } \\
8\end{array}$ \\
\hline Cuidado Infantil & 7.0 & 61 & 12.0 \\
Cargar el agua & 4.8 & 45 & 8.3 \\
Preparación de alimento & 4.8 & 55 & 20.0 \\
limpieza de la casa & 8.3 & 44 & 23.1 \\
\hline
\end{tabular}

(Tomado de: Studies in Family Planning, Vol. 10, Nov. 11/12; Nov./Dic. 1979 pp. $340-41$.

* Cabe resaltar que los clasificados como "otros familiares" juegan, junto con los niños un papel importante en la distribución de las tareas cotidianas.

5 Aquí hacemos referencia al concepto de Burch (1967) que alude más que a la disponibilidad de espacio físico, a la necesidad de reponer miembros con funciones vitales para la familia. 
las mujeres menos calificadas y por tanto con un espectro de opciones muy reducido en términos de las posibilidades de incorporación a la fuerza de trabajo serán las que prioritariamente recurran a la extensión como estrategia de supervivencia. Aquella parte de nuestra subpoblación que efectivamente pueda acceder a ocupaciones remuneradas podrá recurrir a la recomposición siempre y cuando requiera asegurar atención familiar cuando las edades de los hijos así lo demanden. En todo caso la adición de familiares redundará en una acumulación interna de recursos.

Es relativamente frecuente encontrar que las madres viudas, solteras, divorciadas, separadas o en general sin cónyuge pasen, junto con sus dependientes, a formar parte de un núcleo extenso. Dos factores que condicionan la factibilidad de esta opción son la edad de la mujer y el tamaño de su familia (Sweet, 1972). Específicamente, entre las mujeres mayores, las probabilidades de que jefaturen su propia familia aumentan puesto que 1) es factible que su familia de procreación se haya disuelto; 2) sus familias son más grandes que las de las mujeres jóvenes y esto dificulta su asimilación a otros núcleos; 3) pueden tener resueltos sus problemas financieros y de atención familiar; 4) pueden tener hijos ya maduros capaces de asumir las funciones correspondientes al jefe de la familia.

En síntesis, las mujeres mayores no siempre enfrentan la necesidad de reconciliar su papel como trabajadoras y madres, situación que sí se plantea entre las mujeres más jóvenes.

Como en el caso de las mujeres en general, las jefes de familia vinculadas a actividades asalariadas se emplean principalmente en trabajos eventuales y poco redituables. En todo caso, la naturaleza de sus opciones ocupacionales depende, entre otras cosas, de la zona en la que residen. La distinción del lugar de residencia en términos de zonas rurales o urbanas es importante en la medida en que se trata de un factor que afecta tanto la distribución del empleo como la propia estructura familiar. Así, en las zonas urbanas las mujeres se encuentran fundamentalmente vinculadas a actividades tales como comercio ambulante y servicios domésticos (Smith, 1973) en tanto que las habitantes de zonas rurales participan de manera "informal" en la producción agrícola, esto es, cuando el trabajo en la unidad de producción recae en los propios miembros de la familia o bien contratándose en los períodos de zafra.

La inspección de los correlatos de las jefaturas femeninas, que a su vez tienden a intervenir en la formación de núcleos extensos, ciertamente estaría incompleta si no reconociéramos la forma específica en la que las características de la propia unidad familiar influyen en 
la probabilidad de incorporación de familiares ajenos al núcleo. A los efectos de ilustrar la importancia de este factor, podemos pensar que las jefes de familia cuyas unidades nucleares incluyen un número relativamente grande de miembros, se verán menos inclinadas a incorporar parientes extensos a la familia. Asimismo, cuando en la familia nuclear existan miembros capaces de relevar la figura del padre y asumir sus funciones económicas, las posibilidades de incorporación de miembros ajenos, tenderían igualmente a disminuir. Desde luego, la sola presencia de otros familiares en el núcleo no es necesariamente indicativa de que estos sean portadores de ingresos subsidiarios de la familia. La precisión de las funciones que los parientes extensos desempeñan pasa necesariamente por su análisis en tanto que componente diferenciado.

\section{Sobre la muestra}

Este análisis se basa en datos extraídos de una muestra nacional relevada en el Perú en el año 1970 por el Centro Estadístico de Mano de Obra del Servicio Peruano de Empleo y Recursos Humanos. ${ }^{6}$ La muestra, ${ }^{7}$ identificada por las siglas NMSP (National Multipurpose Survey of Perú), comprende información relativa a un total de 5,487 residencias que abarcan 30,360 individuos.

La submuestra que construimos para este trabajo aisló 3,974 unidades familiares, esto es, hogares en los cuales habita una mujer con sus hijos o bien una pareja. Así, de la muestra original se desechó la información relativa a 639 residencias no familiares y a 874 residencias en las que vivía un hombre sólo con su descendencia.

Los datos del NMSP son particularmente adecuados para nuestras consideraciones ya que permiten la utilización de individuos o bien de familias como unidades de análisis y permite la distinción entre hogares urbanos y rurales.

\section{Resultados}

La información recogida en el cuadro 2 (ver anexo) muestra que las mujeres jefes de familia tienden a ser mayores que los hombres, además de que en general tienen un nivel de educación mucho me-

6 El proyecto fue financiado por el Ministerio de Economía y Finanzas y por AID, en tanto que el Centro de Estadística de la Universidad de Michigan participó brindando asesoría técnica.

7 El NMSP es una muestra estratificada y probabilística que cubre un rango muy amplio de información sobre la población y la sociedad peruana. 
nor que el de estos últimos. Estas diferencias son importantes en la medida en que afectan las potencialidades de la mujer en lo que concierne al empleo y al monto de sus ingresos. Es interesante notar que en las ciudades los hombres tienen en promedio 6.5 años de escolaridad en tanto que las mujeres recorren dos años menos. En las áreas rurales las diferencias en la escolaridad atendiendo al sexo del individuo son menores aunque de maỳor significación, ya que en tanto que los hombres tienen en promedio 2 años de escuela, las mujeres son prácticamente todas iletradas.

Por lo que hace a la inserción de los jefes de familia en la fuerza de trabajo, debe señalarse que en ambas áreas residenciales, los hombres tienen índices de participación más altos que las mujeres. El mercado de trabajo en las ciudades es aparentemente más restrictivo en la medida en que solamente un $55 \%$ de las jefes de familia que allí residen participan en la fuerza de trabajo, en tanto que un $81 \%$ de las mujeres rurales trabaja fuera del núcleo familiar.

Las diferencias en el grado de participación, de la población en la fuerza de trabajo en las zonas urbanas y las rurales, tienen que ver con las modalidades que ha asumido en el Perú la expansión capitalista, y por supuesto con la distinta naturaleza de los procesos productivos.

Proponemos así que la tendencia reciente hacia la reducción relativa del empleo agrícola e industrial, así como la creciente masculinización de la fuerza de trabajo en general, han afectado negativamente las posibilidades de incorporación de la mujer a la población económicamente activa.

Aunque la estructura industrial peruana ha experimentado un proceso de expansión productiva a lo largo de las últimas décadas, entre 1940 y 1961 el empleo en el sector manufacturero se contrajo. Adicionalmente, la fuerza de trabajo en su conjunto y particularmente aquélla vinculada al sector industrial se ha masculinizado. Los datos del cuadro 3 permiten constatar que durante este período se registran dos fenómenos importantes: en primer lugar, es notable la escasa proporción en que las mujeres peruanas participan en la fuerza de trabajo; por otra parte, la participación relativa de las mujeres disminuyó entre 1940 y 1961 . Aun en el rubro de servicios, sector en el que las mujeres de otros países tienden a estar sobrerrepresentadas, la composición del empleo no varió; así, la proporción de mujeres vinculadas al sector servicios fue considerablemente más baja que en otrạs naciones de América Latina. 
La inspección de datos más recientes (cuadro 4) muestra lo siguiente:

a) La participación femenina en la fuerza de trabajo permanece más o menos constante durante la década del sesenta.

b) La distribución de la fuerza de trabajo por rama ocupacional ha sufrido modificaciones significativas que reflejan los cambios de la oconomía peruana en su conjunto: mientras que las actividades agrícolas absorbfan a casi $\frac{2}{3}$ partes de la población en 1940, hacia 1972 solamente $\frac{2}{5}$ partes de la fuerza de trabajo se encontraba laborando en la agricultura. Paralelamente, la importancia relativa de los sectores comercio y servicios ha aumentado consistentemente a través de los años. El caso de la manufactura es un tanto particular ya que su importancia relativa entre 1940 y 1961 disminuyó del $15.4 \%$ hasta el 13.3\%. Hacia el principio de los años 70, se aprecia una recuperación importante en el sector que le permite alcanzar altas tasas de crecimiento. $^{8}$

c) Como en otras formaciones sociales, aunque en una proporción relativamente más baja, la mano de obra femenina tiende a concentrarse en las ramas comercio y servicios.

En síntesis, los indicadores de participación de nuestro subgrupo en la fuerza de trabajo reflejan las características peculiares de la economía peruana. En este sentido no resulta sorprendente la constatación de que a pesar de que el rango de opciones ocupacionales en las ciudades es más amplio que el característico de las zonas rurales, las mujeres jefes de familia se encuentran en su mayoría excluidas de aquellos empleos que garantizan estabilidad y altos ingresos. ${ }^{9}$ Sin duda, las características que, en términos de educación, exhiben las mujeres es un factor que también tiene alguna influencia en este fenómeno de exclusión; sin embargo, como muestra el cuadro 2, la variable años de escolaridad no da cuenta de las diferencias observadas en los niveles de empleo considerando la zona de residencia.

En las áreas rurales tanto hombres como, mujeres se encuentran vinculados a las actividades agrícolas aunque cabe observar que una

8 En 1970 el sector crece un $20.7 \%$ y en 1973 un $14 \%$.

9 Las tabulaciones especiales que se hicieron al efecto no se incluyen en este trabajo. Cabe anotar sin embargo que sólo el $5.8 \%$ de las jefes de familia tenían el estatuto de profesionales o técnicos mientras que alrededor del $50 \%$ desempeñaban labores tales como servicio doméstica y pequeño comercio. 
considerable proporción de la población femenina participa asimismo en actividades tales como el pequeño comercio y los servicios de diversa índole.

Desafortunadamente no tenemos información confiable que permita apreciar los niveles de ingresos, ${ }^{10}$ lo que sin duda precisaría en forma más fina el grado de desventaja de las mujeres respecto de los hombres; sin embargo, la evidencia registrada en párrafos anteriores es consistente con las conclusiones a las que han llegado otros estudios (Ross y Sawhill, 1975); Cutright, 1974; Buvinic y Youssef, 1978).

Lo que importa destacar es que las familias encabezadas por una mujer se inclinan más a buscar reestructuraciones en el interior de sus núcleos, tales que compensen la ausencia de un ingreso primario: como se aprecia en el cuadro 2, las familias según estén encabezadas por un hombre o una mujer presentan diferencias en términos de su composición, ya que las últimas tienden a incorporar uno o más miembros no nucleares. Las unidades familiares extensas son más frecuentes en las áreas urbanas que en las rurales aunque las mujeres en una proporción más alta que los hombres, tienden a encabezar familias extensas en ambas áreas de residencia. Aproximadamente la mitad de todos los hogares urbanos encabezados por una mujer contienen por lo menos un miembro no nuclear en tanto que sólo $\frac{1}{3}$ de las familias cuya jefatura es la tradicional incluyen parientes ajenos al núcleo. En las zonas rurales una cuarta parte de los hogares encabezados por varón son de carácter extenso y el $43 \%$ de aquéllos jefaturados por una mujer son del mismo tipo. Independientemente de esto, en promedio las familias encabezadas por una mujer son numéricamente más reducidas que las otras.

Si es válida la propuesta de que el mecanismo de extensión familiar, concebido como una respuesta demográfica frente a la ausencia del cónyuge, contribuye significativamente a incrementar las opciones económicas de la familia, debiera seguirse lógicamente, que las unidades familiares encabezadas por una mujer habrían de presentar una tendencia más pronunciada a la incorporación de parientes no nucleares que aquellas familias jefaturadas en forma tradicional. La proclividad a la formación de familias extensas debiera ser particularmente pronunciada para aquellas mujeres que o no trabajan fuera

10 El relevamiento de la muestra fue financiado por el Ministerio de Economía y Finanzas de la USAID. Una gran cantidad de información sobre la variable ingresos, se perdió al no considerarse formas de pago no monetarias. Tampoco se consideraron flujos de ingreso distintos al principio. En vista de estos problemas no consideramos confiables los datos sobre este aspecto. 
de sus núcleos familiares o bien se encuentran subutilizadas en el mercado de trabajo. Más concretamente, sugerimos que aquellos hogares, en los cuales el jefe de familia no participa con su fuerza de trabajo, presentarán un número mayor de coresidentes no nucleares que los que contendrán los hogares en los cuales el jefe está adecuadamente empleado.

En la medida en que la propensión hacia la extensión se encuentra a su vez limitada por la factibilidad de redistribuir el trabajo en el interior del núcleo, principalmente entre los hijos (Tienda, 1980) y además, considerando que existen también limitaciones de espacio, proponemos que la probabilidad de que un hogar contenga uno o más miembros no nucleares se encuentra inversamente relacionada con el número de trabajadores nucleares que conformen la unidad familiar. Esto es así ya que normalmente los ingresos que permiten la subsistencia del núcleo, provienen en primera instancia de los propios miembros inmediatos (Angel y Tienda, 1980).

Los datos sintetizados en los cuadros 5 y 6 sostienen de alguna manera las propuestas esbozadas anteriormente; sin embargo la evidencia es relativamente confusa y en modo alguno permite aseveraciones causales acerca de las bases de la formación de familias extensas.

El cuadro 5 muestra que el tamaño promedio del componente no nuclear es mayor en los hogares encabezados por una mujer ya sea que la familia habite en zonas rurales o urbanas. Específicamente, las familias urbanas jefaturadas por una mujer contienen, en promedio 1.6 veces más parientes no nucleares que aquéllas jefaturadas en forma tradicional. En las áreas rurales, el tamaño promedio del componente no nuclear es 2.5 veces más grandes en los hogares encabezados por una mujer. Si bien las familias extensas en general son menos frecuentes en las zonas rurales (cuadro 2), vale la pena señalar que el tamaño promedio del componente no nuclear es muy similar en el caso de las familias jefaturadas por una mujer en ambas zonas de residencia: 1.23 y 1.18 personas para áreas urbanas y rurales respectivamente. En contraste, el comportamiento del componente no nuclear entre los hogares jefaturados en forma tradicional varía significativamente según el lugar de residencia.

Como se había previsto, el número promedio de parientes ajenos al núcleo es mayor en aquellos casos en los que el jefe de familia no participa en la fuerza de trabajo. En ambas zonas de residencia, las mujeres jefes de familia que no están vinculadas a la fuerza de trabajo tienen más parientes extensos en sus núcleos que los jefes varones que se encuentran en una situación similar. Encontramos un patrón 
similar entre los jefes subempleados. ${ }^{11}$ y los adecuadamente empleados, ${ }^{12}$ excepto que las diferencias atendiendo al sexo del jefe de familia, en el tamaño del componente no nuclear son mucho más pronunciados para los jefes subempleados que para los adecuadamente empleados. En términos generales, parece configurarse un cuadro que p rrmite afirmar que en efecto, las mujeres son más propensas a la formación de familias extensas, estrictamente por razones de orden económico. No obstante y ya que la evidencia es más sugerente que definitiva, parece razonable pasar ahora a considerar las formas en las cuales la disponibilidad de suficientes parientes nucleares inhibe la tendencia de un núcleo a la incorporación de parientes ajenos a éste.

Si los miembros del núcleo pueden efectivamente convertirse en relevos del padre ausente asumiendo las funciones económicas que a éste corresponden, es razonable esperar que el número de parientes extensos presentes en el hogar varíe en razón inversa al número de trabajadores nucleares de la familia. Los resultados del cuadro 6 proveen evidencia mezclada en apoyo de esta suposición: entre los hogares urbanos jefaturados en forma tradicional, el promedio de parientes extensos aumenta más que disminuye conforme el número de trabajadores nucleares crece. Así, existen muy pocas bases para asumir que las necesidades de ingreso suplementario son el factor que subyace a la formación de núcleos extensos en el caso de las familias encabezadas por la modalidad tradicional. Más bien, la preeminencia de familias extensas en las ciudades peruanas puede imputarse a la intensidad del flujo migratorio campo-ciudad, fenómeno que implica en muchos casos la necesidad de corresidencia con los parientes de la ciudad hasta tanto los migrantes no puedan ubicarse en su propia vivienda y consigan trabajo.

Por otra parte, entre las ianiiiias urbanas encabezadas por una mujer sola, el tamaño del componente no nuclear no varía sistemáticamente en relación al número de trabajadores nuclearss ya que, estadísticamente hablando, el tamaño promedio del componente no nuclear es virtualmente idéntico para las distintas categorías de número de trabajadores nucleares.

La situación es distinta en las zonas rurales, donde aparentemente

11 En esta categoría se incluyen individuos que trabajaron por tiempo parcial o bien que trabajando tiempo completo obtuvieron salarios inferiores al mínimo.

12 Se trata de individuos que formaron parte de la PEA y no fueron subutilizados. Ver Sullivan (1974), para una descripción más detallada de la noción de utilización de la fuerza de trabajo. 
las bases económicas que subyacen a la formación de familias extensas son más pronunciadas.

Es notable que entre las familias rurales que carecen de miembros nucleares vinculados a la fuerza de trabajo, aquéllas jefaturadas por hombrès más que las encabezadas por una mujer incorporan parientes ajenos al núcleo. Esto es extraordinario ya que en condiciones iguales, las unidades de este tipo sin duda tienen una menor facilidad para absorber nuevos miembros y por otra parte, debieran disponer de un margen más amplio para incrementar los ingresos familiares vía el trabajo de la esposa o los hijos adultos. Debe subrayarse, sin embargo, que existe un número relativamente reducido de hogares rurales sin miembros vinculados a la fuerza de trabajo, así pues, la magnitud del diferencial observado atendiendo al tipo de jefatura debe ser contemplado con cierta cautela. Hecha esta excepción, el patrón de relación entre composición familiar extensa y el número de trabajadores nucleares se ajusta a nuestras expectativas. Esto es, en el caso de las unidades encabezadas por una mujer, el tamaño del componente no nuclear varía en razón inversa al número de trabajadores nucleares en tanto que, para las otras familias el patrón de relación es menos sistemático.

Las tabulaciones bivariadas que hemos venido considerando hasta ahora si bien son informativas en cuanto a la composición familiar, no pueden captar la complejidad de diversos factores que influyen en la probabilidad de que un núcleo contenga uno o más miembros ajenos a él. Cuando más son capaces de subrayar algunos de los aspectos claves del proceso mediante el cual los individuos se distribuyen en los hogares. Así pues, en el resto de este trabajo se utiliza un marco multivariado a fin de profundizar en la propuesta original en cuanto a que las mujeres jefes de familia muestran una mayor tendencia a la formación de familias extensas debido a su desventajosa situación socioeconómica respecto de los núcleos jefaturados en forma tradicional. Una posibilidad adicional que ofrece el análisis multivariado estriba en que permite aislar la importancia relativa de las características individuales y las del núcleo en la determinación de la propensión a la extensión.

\section{Análisis multivariado}

Nuestro razonamiento respecto de los factores que determinan la formación de unidades familiares extensas sugiere que la variable educación, concebida como un estimador de la factibilidad de que el jefe de familia vía su incorporación al trabajo asalariado cuente 
con ingresos monetarios, estará en relación inversa con la probabilidad de que la familia contenga uno o más parientes no nucleares. Más aún, esperaríamos que esta relación fuese más pronunciada en las áreas urbanas ya que en éstas la población tiene un nivel promedio de escolaridad más alto que en zonas rurales. Por otra parte y tomando en cuenta nuestras consideraciones anteriores respecto al significado de la participación del jefe de familia en la fuerza de trabajo, proponemos que el comportamiento de esta variable reflejará en forma más directa la motivación económica que subyace a la formación de núcleos extensos.

Se propone que dos aspectos de la estructura familiar, el número de dependientes nucleares y el ciclo de vida familiar, influyen en la probabilidad de formación de la familia extensa. Cuando en el hogar haya un número considerable de dependientes, la presencia de individuos ajenos al núcleo puede coadyuvar al enfrentamiento de las demandas económicas que supone la atención de los dependientes. Sin embargo, en función de la estructura de edades de la familia, los miembros nucleares incluyendo a los dependientes pueden acceder a empleos remunerados de tal suerte que las tensiones económicas queden resueltas en el interior del propio núcleo (Lomnitz, 1976). Esta situación, entonces, mitigaría la necesidad de compartir la carga doméstica y/o la generàción de ingresos complementarios; así, resulta indispensable considerar la influencia simultánea de dos variables: el número de dependientes y el número de trabajadores nucleares presentes en el hogar.

En cuanto al ciclo de vida familiar, se propone que se presenta una mayor propensión a la extensión cuando la familia se encuentra en los primeros y los últimos años de su ciclo. Esto es así porque las familias que no han empezado o que recién empiezan a expandirse vía la fertilidad así como aquéllas que empiezan a contraerse debido a la pérdida de miembros nucleares en edad adulta o bien a la muerte del cónyuge requieren, en el primer caso, compartir responsabilidades $y$ en el segundo ingresos suplementarios puesto que las mujeres de mayor edad se enfrentan más desventajosamente al mercado de trabajo. A menos que en el núcleo se cuente con la presencia de descendientes en edad adulta capaces de asumir las funciones de la jefatura familiar, la mujer sin cónyuge tendrá una mayor inclinación a recomponer su familia.

A fin de operacionalizar la noción de ciclo de vida familiar, proponemos la siguiente tipología (Duvall, 1957):

1. Familias de reciente creación: aquéllas en las que no hay descendencia y en las que la madre tiene menos de 45 años. 
2. Familias en etapa de procreación: aquéllas en las que el hijo mayor tiene menos de 6 años.

3. Familias en etapa escolar: aquéllas en las que el hijo mayor tiene entre 6 y 13 años.

4. Familias en etapa adolescente: aquéllas en las que el hijo mayor tiene entre 14 y 18 años.

. Familias con descendencia adulta: aquéllas en las que el hijo mayor tiene 19 años o más.

6. Familias vacías: cuando ya no hay descendencia en el hogar y la madre tiene más de 45 años.

Estas seis categorías reflejan varias consideraciones tanto teóricas como empíricas, entre las cuales cabe subrayar: los límites de edad del estatuto de familiar dependiente, las nociones de los teóricos del desarrollo familiar respecto de los eventos clave por los que pasa toda familia y la idea de que la descendencia puede considerarse adulta a partir de los 19 años.

La técnica de análisis multivariado utilizada para estimar la incidencia de los efectos combinados de las características sociodemográficas del jefe de familia y de su núcleo en la probabilidad de que la familia sea de tipo extenso es un análisis de clasificación múltiple. Los efectos brutos se expresan como desviaciones respecto del total de la muestra compuesta por familias extensas (i.e., la media de la variable dependiente) y se interpretan como diferencias porcentuales. Los efectos netos son coeficientes parciales de regresión ajustados considerando los efectos producidos por las otras variables incluidas en la ecuación. La importancia relativa de cada factor se indica utilizando los índices Beta, mismos que se construyen en forma análoga a los coeficientes de regresión estandarizados.

El cuadro 7 muestra los resultados del análisis de clasificación múltiple (MCA). Antes de comentar los resultados más específicos conviene subrayar que las circunstancias que conducen a la formación de núcleos extensos son distintas según se trate d" familias urbanas o rurales. Esto es evidente, no sólo tomando en cuenta las variaciones en el poder explicativo del modelo utilinado para las cuatro subpoblaciones consideradas, sino tambié.l otservando que el patrón de relación entre la variable dependiente y las independientes es disímil entre los grupos. En general, el modelo es más adecuado para 
analizar el caso de las familias extensas en áreas rurales. Particularmente, para el caso de las familias encabezadas por una mujer, las 5 variables utilizadas explican el $28 \%$ de la varianza, en tanto que para los hogares urbanos jefaturados en forma tradicional solamente se da cuenta de un $5 \%$. Esto último puede deberse en parte a que el modelo no considera el fenómeno de la migración interna como uno de los determinantes de la formación de núcleos extensos en las ciudades (Walker y Gendell, 1976). Sin embargo, debe tomarse en cuenta que el modelo tiene un mayor poder de explicación para el caso de los hogares encabezados por una mujer, por lo que puede pensarse en la validez relativa del mismo.

De mayor interés resultan los patrones de asociación y la magnitud de los efectos de cada factor comprendido en el modelo. La hipótesis de la existencia de una relación inversa entre nivel de educación y composición extensa se confirma para el caso de los hogares jefaturados en forma tradicional, sin embargo hay alguna evidencia de que la relación no es lineal. La magnitud de las betas parciales indican que la variable educación es relativamente más importante para el caso de los jefes varones que habitan en las ciudades. Aparentemente el efecto del nivel de escolaridad entre las jefes mujeres es insignificante, resultado que refleja parcialmente el hecho de que las mujeres con altos niveles de escolaridad tienden, en menor proporción, a la disrupción familiar y por lo tanto a jefaturar sus propias familias. Asimismo, habida cuenta de los bajos niveles de escolaridad característica de los jefes de familia mujeres, las diferencias en cuanto a posibilidades de ingreso son insuficientes (ver cuadro 7).

La variable participación en la fuerza de trabajo, se encuentra vinculada con la tendencia a la composición familiar extensa solamente para el caso de hogares rurales jefaturados por un varón. Como habíamos anticipado, los jefes de familia desempleados en general tienden más a incorporar familiares ajenos al núcleo en comparación con aquéllos que están adecuadamente empleados, pero la diferencia entre las dos categorías es bastante reducida (solamente $8 \%$ ). Por otra parte, los jefes de familia subempleados se inclinan un $28 \%$ menos a incorporar uno o más parientes no nucleares a sus hogares que aquéllos que están desempleados. Entre el subgrupo de familias rurales jefaturadas por una mujer, el patrón de relación entre la variable participación del jefe de familia en la fuerza de trabajo y la variable dependiente se ajusta a lo esperado y los efectos netos están muy cerca de la significación estadística.

Los efectos de la variable ciclo de vida familiar sobre la propensión a la extensión son, a juzgar por la magnitud de las betas parcia- 
les, los más pronunciados. Para el caso de hogares urbanos encabezados por una mujer, esta variable es la única que incide significativamente en la variable dependiente. En las áreas urbanas, el $88 \%$ de todas las familias encabezadas por una mujer que se encuentran en la etapa de procreación contienen uno o más parientes ajenos al núcleo, en tanto que solamente un $38 \%$ de aquéllas en las etapas escolar y adolescente son de tipo extenso. Este patrón de relación obra en apoyo de la noción de que las familias que se encuentran en sus fases picos de expansión se inclinan menos a la incorporación de otros miembros debido a que pueden redistribuir más flexiblemente las cargas domésticas. Esta opción está prácticamente cerrada para el caso de aquellas mujeres cuyo hijo mayor tiene menos de 6 años, aquí, la necesidad de recurrir al mecanismo de extensión es más significativa.

Los hogares rurales encabezados por una mujer presentan una forma de relación distinta entre el ciclo de vida familiar y la extensión. Específicamente, los hogares en etapa preescolar tienen un $17 \%$ menos de probabilidades de ser de tipo extenso. Sin embargo, aquéllos donde el hijo mayor está en la edad adulta tienden un 11\% más a incluir parientes ajenos al núcleo. Debemos considerar al menos dos circunstancias que en parte podrían explicar estas diferencias entre los hogares urbanos y rurales jefaturados por mujeres.

En primer lugar, en las áreas rurales los lazos familiares son susceptibles de mantenerse sin el requisito de la corresidencia. Las mujeres con hijos pequeños pueden recurrir a sus parientes en busca de asistencia económica y servicio doméstico. Asimismo, en las zonas rurales la persistencia de economías domésticas tendería a reducir la necesidad de los jefes de familia de recurrir a los corresidentes para que éstos, a través de su participación en la fuerza de trabajo, complementaran los ingresos monetarios de la familia. Estas circunstancias no se presentan en las zonas urbanas, en las cuales la mujer que tiene una ocupación remunerada normalmente se enfrenta a horarios y rutinas más rígidas.

En segundo lugar, la mayor propensión a la extensión característica de las últimas etapas de la vida familiar puede explicarse por la necesidad de las mujeres mayores y generalmente viudas de asegurarse recursos para sostener su vejez a través de sus hijos casados.

Los efectos del ciclo de vida para el caso de los hogares jefaturados en forma tradicional son mucho menores comparativamente, aunque presentan tendencias similares.

A la luz de los resultados del cuadro 6, no resulta extraño que el número de trabajadores del núcleo no esté sistemáticamente relacionado con la probabilidad de que las familias encabezadas por una mu- 
jer contengan miembros adicionales. Solamente entre los hogares sitos en áreas rurales y jefaturados por una mujer puede apreciarse una relación estadísticamente significativa entre las variables. Por lo demás, aunque en comparación con los factores "nivel de escolaridad" y "participación en la fuerza de trabajo", esta variable tiene un peso sustancial, el patrón de la relación no es sistemático. En los hogares en los que no hay trabajadores nucleares tiende a haber menos familiares ajenos al núcleo que en aquéllos en los que hay 3 o más personas económicamente activas. Estos últimos se inclinan un $43 \%$ más a contener uno o más parientes adicionales $(-.30-.13=.43)$. El resultado puede explicarse en parte por el hecho de que en las áreas rurales, donde la subutilización de la fuerza de trabajo es bastante considerable, un número alto de miembros económicamente activos frecuentemente expresan la incapacidad de otros sectores para absorber esta fuerza de trabajo y por otra parte constituye una estrategia viable para enfrentar el desempleo. En estos casos, los parientes nucleares, particularmente los adultos económicamente activos efectivamente contribuían a hacer viable la unidad doméstica. Esta interpretación es apenas tentativa y requerida de más abundamiento; por ejemplo, sería importante detenerse en el análisis de las características sociodemográficas de aquellas familias en las que no existen miembros económicamente activos.

La distribución por edades de los miembros de la familia (en este caso determinada por la vía de la especificación del número de hijos que dependen económicamente del jefe de familia) parece ser un factor sustancial en la determinación de la probabilidad de que una familia rural sea extensa; sin embargo, esto no se aplica a las familias urbanas. En el caso de las familias rurales jefaturadas por una mujer, el número de dependientes nucleares que residen en el hogar influye en la probabilidad de que otros parientes sean parte de la familia. Contrariamente a lo esperado, los hogares en los que no hay dependientes tienden más a ser de tipo extenso que aquéllos en los que hay 3 o más dependientes. Específicamente, mientras que cerca del $54 \%$ del total de las unidades familiares rurales encabezadas por una mujer en las que no hay dependientes contienen uno o más miembros no nucleares, soiamente un $15 \%$ de los que contienen entre 3 y 4 dependientes son familias extensas. En el caso de las familias jefaturadas en forma tradicional, se observa un patrón de relación similar aunque las medias para la categoría son menores. Vale la pena notar que existe un patrón de relación ligeramente no lineal entre composición extensa y la presencia de varios dependientes para los dos tipos de jefatura. 


\section{Nota final}

En síntesis, estos resultados muestran que los factores que influyen en la probabilidad de formación de familias extensas varían según el lugar de residencia y el tipo de jefatura familiar. En ambas zonas, las mujeres jefes de familia presentaron una mayor tendencia hacia la recomposición de sus núcleos. Así, no solamente es mayor la incidencia de familias extensas entre las unidades jefaturadas por una mujer sino que además el tamaño promedio del componente no nuclear es consistentemente más grande cuando se le compara con el caso de las familias jefaturadas en forma tradicional. Los resultados del análisis multivariado son, sin embargo, más sugerentes que definitivos en cuanto a la racionalidad económica del mecanismo de extensión.

Sintetizando, el nivel de educación del jefe de familia y el ciclo de vida familiar resultaron estims lores significativos de la probabilidad de que la familia sea extınsa para el caso de las unidades urbanas donde el jefe es un hombre. Pero en el caso de las familias jefaturadas por una mujer sólo la última variable está significativamente relacionada con la composición familiar extensa. Es posible que algunas variables que no se consideraron en el modelo tales como el estatus de migrante del jefe o la disponibilidad de parientes en lugares cercanos, sean factores que contribuyan más a la explicación de la forma que asume la composición familiar extensa 'n las urbes peruanas. Estas variables no pudieron ser examinadas directamente con nuestros datos, sin embargo el trabajo de Roberts (1976) es muy sugerente al respecto.

En cuanto a las áreas rurales puede observarse que los aspectos demográficos de la composición familiar juegan un papel significativo en la propensión a la extensión familiar. Para ambos tipos de jetura, las variables "ciclo de vida familiar" y "número de dependientes nucleares" influyen significativamente en la probabilidad de que la familia contenga miembros no nucleares. Por otra parte, la importancia de la participación del jefe de familia en la fuerza de trabajo, en la determinación de la composición extensa, varía según se trate de unidades jefaturadas por hombres o mujeres. En el caso de las unidades jefaturadas por hombres, la participación del jefe en la fuerza de trabajo influye en la probabilidad de que la familia sea extensa, mientras que entre las mujeres, es la presencia de miembros adicionales vinculados a la fuerza de trabajo el factor que incide sobre la probabilidad de que la familia contenga otros parientes.

Nuestra tesis básica respecto a que las mujeres jefes de familia se inclinan más a la recomposición de sus núcleos para restablecer en 
parte la ausencia del padre, queda en general demostrada. En tanto que esta respuesta demográfica puede en efecto tener una cierta racionalidad económica, en el fondo refleja más que eso. El pronunciado efect; 'sel ciclo de vida familiar sugiere no sólo el hecho de que las mujures mayores se encuentran más frecuentemente sin cónyuges sino también que disponen de más "espacios vitales" que suplir. Estas circunstancias son consistentes y se retroalimentan mutuamente.

En la medida en que las variables nivel educativo y participación en la fuerza de trabajo no ejercen una influencia significativa en la propensión que presentan las familias jefaturadas por una mujer a la extensión, parece necesario matizar el peso de la determinación económica del fenómeno. Sin embargo, sería prematuro descalificar por completo la lógica de nuestra argumentación. En las áreas rurales, por ejemplo, la presencia de dependientes así como la disponibilidad de parientes nucleares capaces de generar ingresos influyen significativamente en la probabilidad de que una mujer jefe de familia reestructure su núcleo. Por otra parte, nuestro análisis de las variables económicas se vio limitado por la ausencia de datos fidedignos sobre rangos de ingreso familiar. Una evaluación más rigurosa del argumento económico requeriría el análisis de los intercambios monetarios y no monetarios entre los familiares de los núcleos a fin de determinar la forma en que éstos contribuyen a la supervivencia de la familia.

Por último conviene destacar que este análisis se ha circunscrito a los patrones de corresidencia dejando de lado las redes de asistencia familiar. Tanto los patrones de corresidencia como las redes familiares que no implican necesariamente la formación de núcleos extensos son pertinentes en la investigación de las estrategias de supervivencia familiar. En este sentido, 1.s datos estadísticos no pueden sustituir a los relevamientos de campo en los que se puede acentuar la información más cualitativa. 


\title{
ANEXO
}

\author{
Cuadro 2
}

CARACTERÍSTICAS SOCIALES Y DEMOGRÁFICAS DE LOS NÚCLEOS FAMILIARES PERUANOS, POR TIPO DE JEFATURA FAMILIAR Y LUGAR DE RESIDENCIA

\begin{tabular}{|c|c|c|c|c|}
\hline \multirow{3}{*}{$\begin{array}{l}\text { Caracteristicas del jefe } \\
\text { de familia y del nucleo }\end{array}$} & \multicolumn{4}{|c|}{ Lugar de residencia y tipo de jefatura familiar } \\
\hline & \multicolumn{2}{|c|}{ urbana } & \multicolumn{2}{|c|}{ rural } \\
\hline & $\begin{array}{l}\text { jefatura } \\
\text { tradicional }\end{array}$ & $\begin{array}{l}\text { jefatura } \\
\text { femenina }\end{array}$ & $\underset{\text { jefatura }}{\text { jedicional }}$ & $\begin{array}{l}\text { jefatura } \\
\text { femenina }\end{array}$ \\
\hline \multicolumn{5}{|l|}{ edad $\underline{a} /$} \\
\hline $\begin{array}{l}16=25 \\
26=35 \\
36=45 \\
46=55 \\
56 \text { - mas }\end{array}$ & $\begin{array}{l}7.8 \\
28.3 \\
30.2 \\
20.0 \\
13.8\end{array}$ & $\begin{array}{l}3.5 \\
20.3 \\
30.5 \\
20.8 \\
24.9\end{array}$ & $\begin{array}{r}8.9 \\
22.2 \\
28.8 \\
19.1 \\
21.0\end{array}$ & $\begin{array}{r}3.1 \\
13.6 \\
30.4 \\
22.0 \\
30.9\end{array}$ \\
\hline educacion $\mathrm{a} /$ & 6.5 & 4.4 & 2.1 & 0.6 \\
\hline $\begin{array}{l}\text { Tasa de participacion en } \\
\text { la fuerza de trabajo b/ }\end{array}$ & 93.1 & 55.5 & 98.1 & 80.7 \\
\hline $\begin{array}{l}\text { Namero de familias exten } \\
\text { sas en relacion al totaI }\end{array}$ & 34.2 & 48.0 & 25.7 & 42.7 \\
\hline $\begin{array}{l}\text { Tamaño del nácleob/ } \underline{c} / \\
2 \\
\begin{array}{l}3=4 \\
5-6 \\
7 \text { o mas }\end{array}\end{array}$ & $\begin{array}{r}5.8 \\
26.3 \\
31.2 \\
36.8\end{array}$ & $\begin{array}{l}11.0 \\
33.6 \\
27.6 \\
27.7\end{array}$ & $\begin{array}{r}6.9 \\
29.4 \\
34.0 \\
29.7\end{array}$ & $\begin{array}{l}12.0 \\
36.4 \\
39.1 \\
12.5\end{array}$ \\
\hline
\end{tabular}

Fuente: NMSP.

* Familia encabezada por un hombre cuya esposa vive en el hogar.

a En años.

b En porcentaje.

c Basado en todos los parientes corresidentes. 
Cuadro 3

\section{DISTRIBUCIÓN DE LA FUERZA DE TRABAJO POR SEXO Y RAMA DE OCUPACIÓN: PERÚ, 1940 Y 1961}

(en porcentaje)

\begin{tabular}{lrrrrr}
\hline & \multicolumn{2}{c}{ Distribucion } & \multicolumn{2}{c}{ Porcentaje de hombres } \\
Sector & 1940 & 1961 & 1940 & 1961 \\
\hline agricultura & 62.4 & & 50.3 & 68.5 & 86.2 \\
extraccion & 1.8 & 2.2 & 97.3 & 97.3 \\
manufactura & 15.4 & 13.3 & 43.5 & 71.8 \\
construccion & 1.9 & 3.4 & 98.0 & 99.0 \\
comercio & 4.6 & 9.1 & 67.8 & 72.1 \\
transporte $y$ & 2.1 & 3.0 & 95.3 & 95.1 \\
comunicacion & 10.2 & 15.2 & 50.9 & 56.8 \\
servicios & 1.6 & 3.3 & 80.1 & 72.9 \\
otro & 100.0 & 100.0 & 64.6 & 78.4 \\
total & & & & \\
\hline
\end{tabular}

Fuente: Dirección Nacional de Estadística, Censo de Población y Ocupación, 1946 (Lima: 1944), Vol. I, pp. 370, 606-607. Censo Nacional de Población, Características Económicas, 1961.

Cuadro 4

DISTRIBUCIÓN DE LA FUERZA DE TRABAJO POR SEXO Y RAMA DE OCUPACIÓN:

PERÚ, 1972

\begin{tabular}{lccr}
\hline Sector & Distribucion & Hombres & Mujeres \\
\hline agricultura & 43.0 & 89.3 & 10.7 \\
pesca & 1.0 & 98.6 & 1.4 \\
extraccion & 1.5 & 97.4 & 2.7 \\
manufactura & 13.0 & 63.5 & 36.5 \\
electricidad, gas, agua & 0.5 & 94.8 & 5.2 \\
construccion & 5.0 & 99.0 & 1.0 \\
conercio & 11.0 & 53.8 & 46.2 \\
transporte y comunicaciones & 5.0 & 95.7 & 4.3 \\
servicios & 20.0 & 27.3 & 72.7 \\
total & 100.0 & 80.0 & 20.0 \\
\hline
\end{tabular}

Fuente: Dirección Nacional de Estadística, Censo de Población y Ocupación, 1972 (Lima, 1976), Vol. I, cuadros 26 y 37 (pp. 600-764). 
Cuadro 5

NÚMERO PROMEDIO DE PARIENTES EXTENSOS PRESENTES EN EL NÚCLEO, POR PARTICIPACIÓN DEL JEFE DE FAMILIA EN LA FUERZA DE TRABAJO, TIPO DE JEFATURA Y LUGAR DE RESIDENCIA

\begin{tabular}{|c|c|c|c|c|}
\hline \multirow{3}{*}{$\begin{array}{l}\text { Participación en la } \\
\text { fuerza de trabajo }\end{array}$} & \multicolumn{4}{|c|}{ Lugar de residencia y tipo de jefatura } \\
\hline & \multicolumn{2}{|c|}{ urbana } & \multicolumn{2}{|c|}{ rural } \\
\hline & $\underset{\text { tradicional }}{\text { jefatura }}$ & $\begin{array}{l}\text { jefatura } \\
\text { femenina }\end{array}$ & $\begin{array}{c}\text { jefatura } \\
\text { tradicional }\end{array}$ & $\begin{array}{l}\text { jefatura } \\
\text { femenina }\end{array}$ \\
\hline $\begin{array}{l}\text { fuera de la fuerza de } \\
\text { trabajo }\end{array}$ & 1.38 & 1.54 & 1.43 & 1.89 \\
\hline desempleado & 1.11 & $--\underline{a}$ & $--\underline{b} /$ & $--\underline{b} /$ \\
\hline subempleado & 0.68 & 1.02 & 0.46 & 1.02 \\
\hline adecuadamente empleado & 0.72 & 0.93 & 0.75 & 0.99 \\
\hline$t \circ t a 1$ & 0.76 c $/$ & $1.23 \mathrm{e} /$ & $0.49 \mathrm{c} /$ & $1.18 \mathrm{~d} /$ \\
\hline$(\mathrm{N})$ & $(1,742)$ & (297) & $(1,670)$ & (265) \\
\hline
\end{tabular}

Fuente: NMSP

a El tamaño de la celda hace que las estimaciones sean poco confiables.

b Ninguna observación en esta categoría.

c Las medias de la categoría son significativamente distintas, p. $<.001$

d Las medias de la categoría son significativamente distintas, p. $<.01$

e Las medias de la categoría no son significativamente distintas.

Cuadro 6

NÚMERO PROMEDIO DE PARIENTES EXTENSOS PRESENTES EN EL NÚCLEO, POR NÚMERO DE TRABAJADORES NUCLEARES, TIPQ DE JEFATURA FAMILIAR Y LUGAR DE RESIDENCIA

\begin{tabular}{|c|c|c|c|c|c|}
\hline \multirow{2}{*}{\multicolumn{2}{|c|}{$\begin{array}{c}\text { Numero de trabajadores } \\
\text { Nucleares }\end{array}$}} & \multicolumn{4}{|c|}{ Lugar de residencia y tipo de jefatura } \\
\hline & & $\underset{\text { jefatura }}{\mathbf{u} r}$ & $\begin{array}{l}\text { n a } \\
\text { jefatura } \\
\text { femenina }\end{array}$ & $\underset{\text { jefatura }}{r}$ & $\begin{array}{l}\text { a } 1 \\
\text { jefatura } \\
\text { femenina }\end{array}$ \\
\hline & 0 & 0.60 & 1.60 & 1.64 & 0.48 \\
\hline & 1 & 0.05 & 1.13 & 0.38 & 1.81 \\
\hline & 2 & 0.82 & 0.96 & 0.55 & 0.93 \\
\hline & 3 o más & 1.20 & 1.46 & 0.47 & 0.78 \\
\hline total & 11 & $0.76 \mathrm{~b} /$ & $1.23 \mathrm{c} /$ & 0.49 a/ & $1.18^{\mathrm{a}}$ / \\
\hline & $(\mathrm{N})$ & $(1.742)$ & (297) & $(1,670)$ & $(265)$ \\
\hline
\end{tabular}

Fuente: NMSP

a Las medias de la categoría son significativamente distintas, P .001

b Las medias de la categoría son significativamente distintas, $P=.002$

c Las medias de la categoría no son significativamente distintas. 


\section{Cuadro 7}

ANÁLISIS DE CLASIFICACIÓN MÚLTIPLE DE LA PROPENSIÓN A LA FORMACIÓN DE NÚCLEOS EXTENSOS ENTRE FAMILIAS JEFATURADAS POR UNA MUJER Y EN FORMA TRADICIONAL, POR LUGAR DE RESIDENCIA: PERÚ, 1970

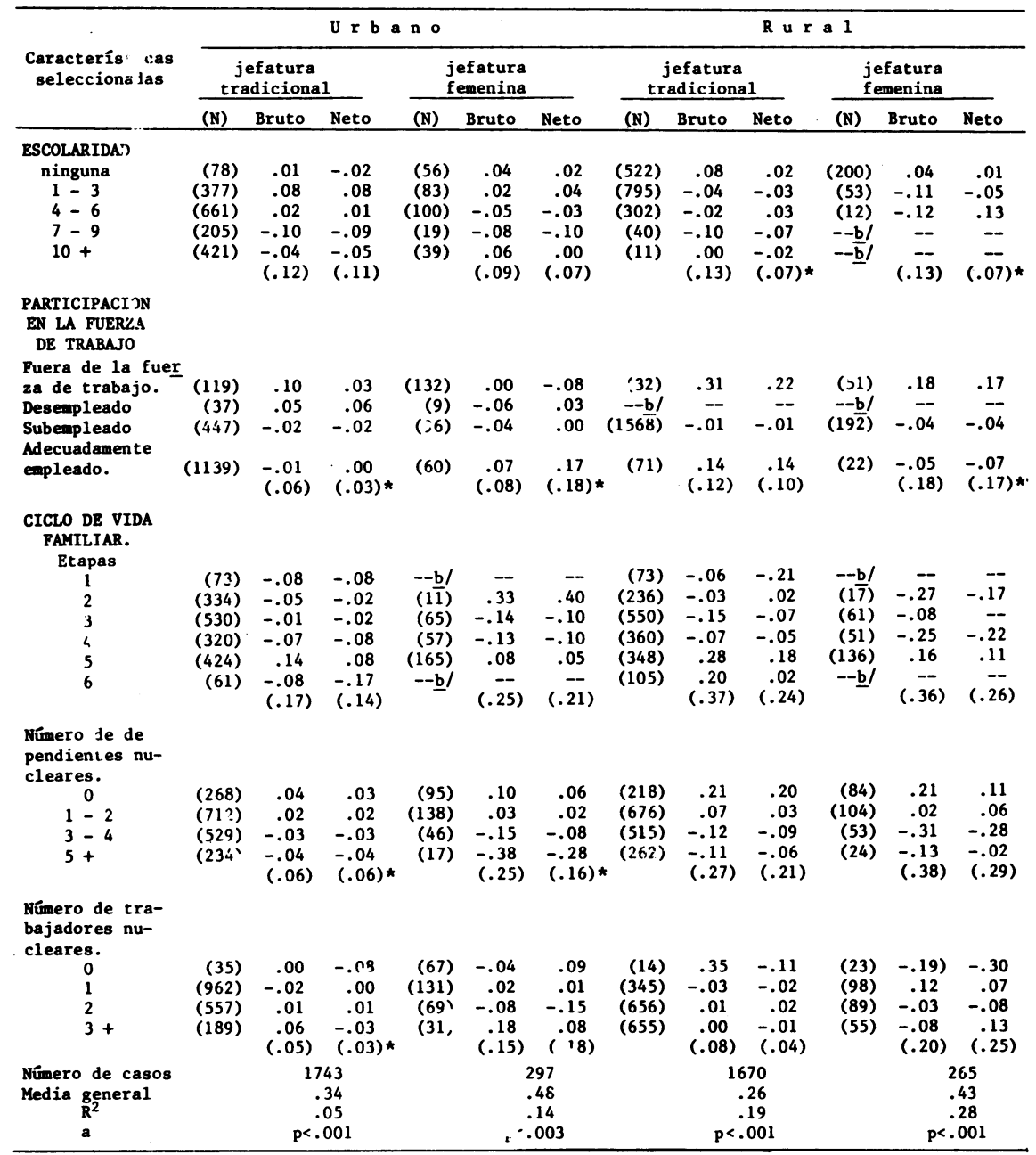

Fuente: NMSP

* No es significativo en $p<.05$

a Ajustado por edad

b Número de observaciones en la celda

c Conforme a los criterios definidos en el texto 


\section{BIBLIOGRAFIA}

Angel, Ronald y Marta Tienda, "Household Composition and Income Generation Strategies Among Anglos, Blacks and Hispanic Origin Groups in the U. S.". "Family and Work Roles in Comparative Perspective", que se realizó en Calgary, Alberta, Canadá, marzo 13-14, 1980.

Blumberg, R. L. y P. García, "The Political Economy of the Motherchild Family: A Cross Societal View”. pp. 99-163 en Luis LeñeroOtero (ed.), Beyond the Nuclear Family Model. London: Sage, 1974.

Buvinic, Mayra y Nadia H. Youssef, "Women-Headed Households: The Ignored Factor in Development Planning", con Barbara Von Elm. Informe presentado a AID/WID, Washington, D. C., marzo, 1978.

Chaney, Elsa y Marianne Schmink, "Las mujeres y la modernización". En Carmen Elú de Leñero (ed.), La mujer en América Latina. México. Editorial Sepsetentas, 1975.

Cohen, Jacob y Patricia Cohen, Applied Multiple Regression/Correlation Analysis for the Behavioral Sciences. New York: Wiley, 1975.

Cooney, Rosemary S., "Demographic Components of Growth in White, Black and Puerto Rican Female Headed Families: A comparison of the Cutright and Ross/Sawhill Methodologies". Social Science Research 8: 144-158, 1979.

Cutright, Phillips, "Components of Change in the Number of Female Family Heads Aged 15-44: United States, 1940-1970”. Journal of Marriage and the Family 36: 714-721, 1974.

Duncan, Greg J., "Unmarried heads of households and marriage". En Duncan and Morgan (eds.), Five Thousand American Families: Patterns of Economic Progress, Vol. IV Chapter III. Ann Arbor: Survey Research Center, The University of Michigan, 1976.

Duvall, Evelyn M., Family Development. Chicago: Lippincott, 1957.

Lomnitz, Larissa, ¿Cómo sobreviven los marginados? México: Siglo XXI Eds., 1975.

"Migration and Network in Latin America". En Alejandro Portes y Harley L. Brownings (eds.), Current Perspectives in Latin American Urban Research. Austin: The University of Texas Press, 1976.

Merrick, Thomas W., "Household Structure and Proverty in Families Headed by Women: The Case of Belo Horizonte". Trabajo presentado en las reuniones conjuntas del Latin American Studies 
Association y the African Studies Association, Houston, noviembre, 1977.

Quijano, Aníbal, "Redefinición de la Dependencia y proceso de marginalización en América Latina". CEPAL (mimeo), 1970.

Roberts, Bryan, "The Provincial Urban System and the Process of Dependency": En Alejandro Portes y Harley L. Brownings (eds.), Current Perspectives in Latin American Urban Research. Austin: The University of Texas Press, 1976.

Ross, Heather L. e Isabel V. Sawhill, Time of Transition: The Growth of Families Headed by Women. Washington, D. C.: The Urban Institute, 1975.

Singelmann, Joachim y Marta Tienda, "Changes in Industry Structure and Female Employment in Latin America. 1950-1970". Sociology and Social Research 63: 745-771, 1979.

Smith, Margo, "Domestic Service as a Channel of Upward Mobility for the Lower Class Woman: The Lima Case". Pp. 191-207 en Snn Pescatello (ed.), Female and Male in Latin America. Pittsburgh: The University of Pittsburgh Press, 1973.

Stinner, William F., Melina Bacol-Montilla y Héctor Morada, "Demographic and Socioeconomic Factors Affecting Residential Arrangements of Young Unmarried Mothers in the Philippines". Trabajo presentado en la Reunión Anual de la Population Association of America, Denver, abril 10-12, 1980.

Sullivan, Teresa, "New Approaches to Labor Force Measurement: Uses for Census Data". Asian and Pacific Census, Newsletter 1: 5-8, 1974.

Sweet, J. A., "The Living Arrangements of Separated, Widowed and Divorced Mothers". Demography 9: 143-157, 1972.

Tienda, Martha, "Child and Spouse Replacement Mechanisms: A Life Cycle Perspective on Family Composition in Peru". International Journal of Sociology of the Family 10: (en prensa), 1980.

Walker, Ann Hamilton y Murray Gendell, "The Relationship of Family Life Cycle and Rural/Urban Residence to Family Size and Composition: Guatemala, 1964". Trabajo presentado en la Reunión Anual de la Population Association of America, Montreal, Canadá, 1976. 